\title{
ARTIBISE, Alan, Winnipeg, An Illustrated History. Coll. « The History of Canadian Cities ». Lorimer \& Company, National Museum of Man, National Museums of Canada, Toronto, 1977. 224 p., ill., index. \$12.95 relié.
}

\section{Jeannine Pozzo et Jacques Bureau}

Volume 32, numéro 4, mars 1979

URI : https://id.erudit.org/iderudit/303731ar

DOI : https://doi.org/10.7202/303731ar

Aller au sommaire du numéro

Éditeur(s)

Institut d'histoire de l'Amérique française

ISSN

0035-2357 (imprimé)

1492-1383 (numérique)

Découvrir la revue

Citer ce compte rendu

Pozzo, J. \& Bureau, J. (1979). Compte rendu de [ARTIBISE, Alan, Winnipeg, An Illustrated History. Coll. " The History of Canadian Cities ». Lorimer \&

Company, National Museum of Man, National Museums of Canada, Toronto, 1977. 224 p., ill., index. \$12.95 relié.] Revue d'histoire de l'Amérique française, 32(4), 629-630. https://doi.org/10.7202/303731ar d'utilisation que vous pouvez consulter en ligne. 


\title{
COMPTES RENDUS
}

\author{
Artibise, Alan, Winnipeg, An Illustrated History. Lorimer \& Com- \\ pany, National Museum of Man, National Museums of Canada, \\ Toronto, 1977, 224 p., ill., index. (coll. "The History of Cana- \\ dian Cities»). \$12.95 relié
}

Importante initiative que celle du Musée national de l'homme à Ottawa: une série de livres traitant du développement urbain au Canada à travers l'histoire de différentes communautés, telles Montréal, Vancouver, Chicoutimi, etc. Pour le premier volume de la série, Alan Artibise a réalisé une histoire illustrée de Winnipeg fort intéressante. L'auteur, qui n'en est pas à son premier essai sur la ville de Winnipeg, décrit l'évolution de la cité de 1812 à 1970 . Les divisions chronologiques du livre se rattachent directement à des phases propres au développement de la ville.

L'introduction situe les débuts d'un établissement modeste, à la jonction des rivières Rouge et Assiniboine, encore ni village, ni ville, portant le nom de Fort Garry (1812-1870). L'histoire de Winnipeg en tant que communauté distincte commence en 1874 ; elle est évoquée dans les trois chapitres constituant le livre: les années de formation et de développement (1874-1913), la crise et le déclin (1914-1945), la transformation et le défi actuel (1946-1970).

En résumé, Winnipeg est d'abord un poste de traite des fourrures, ensuite une ville qui, jusqu'en 1913, connaît une expansion très rapide. À partir de 1919 commence une période de déclin qui ne se terminera qu'après la seconde guerre mondiale, alors que Winnipeg deviendra l'une des plus importantes ville de l'Ouest canadien.

L'expansion économique qui caractérise les 40 premières années de Winnipeg est intimement liée à l'arrivée massive d'immigrants: d'abord des anglophones de l'Ontario qui prennent la tête de la ville et qui essaient, en particulier par l'école, d'assimiler les autres immigrants, comme les Slaves. L'entre-deux-guerres est une période difficile, marquée notamment par la grève de 1919 qui en plus de traduire les difficultés économiques de la ville, exaspère les querelles ethniques non réglées qui se feront sentir jusqu'à la fin de la seconde guerre mondiale. À partir des années 50, un essor économique lié à un équilibre démographique permet de surmonter ces difficultés et favorise l'émergence d'une vie communautaire authentique. 
L'auteur a choisi une centaine de photographies pour illustrer les éléments les plus significatifs de son étude historique. Ces photos, en plus de se référer directement au texte, sont pour la plupart accompagnées d'une légende qui permet de faire valoir divers événements importants de la vie à Winnipeg. De plus, ce livre comprend de nombreux tableaux statistiques concernant surtout le développement et l'origine de la population, des cartes des quartiers de la ville à différentes époques, un index et une bibliographie riche et récente sur la ville de Winnipeg.

L'auteur a réussi à atteindre les objectifs de la série: offrir un volume pouvant intéresser le grand public et les spécialistes de la vie urbaine.

Histoire

Université Laval

Jeannine Po/ro

Jacques Bureau 